\title{
Surgical treatment of a primary tumour and liver metastases in a patient with intracranial haemangiopericytoma: report of a case
}

\author{
Kilda J. Carpenter, BA \\ Medical Sciences Division, University of Oxford, John Radcliffe Hospital, Headley Way, Oxford, OX3 9DU \\ David Nasralla, BMBCh, DPhil, FRCS \\ Nuffield Department of Surgical Sciences, University of Oxford
}

\begin{abstract}
Key Learning Points / Commentary
Cancers exhibit a spectrum of behaviours ranging from aggressive malignancy to those which progress in an almost benign fashion. An appreciation of this aspect of tumour biology is crucial when planning patient management as non-curative debulking procedures for slower growing tumours often still confer a survival benefit. The present report is a case in point.
\end{abstract}

Haemangiopericytoma is a rare cancer whilst clear indications for an ALPPS procedure are still evolving. Inherent to the patient groups in whom ALPPS is considered is the presence of advanced underlying disease. Many of these patients would previously not be considered for liver resection or have progressed beyond resectability by the time sufficient hypertrophy of their future liver remnant had been achieved using conventional two-stage hepatectomy techniques.

ALPPS offers another treatment option for these patients, although at a cost. Early experience reported unacceptably high morbidity and mortality rates following ALPPs resection and suggested that tumour type and patient fitness were key factors in determining outcome. The patient must be fit enough to withstand two major operations 1-2 weeks apart. The slow growing nature of the tumour in the present case combined with the fit,

Keywords: ALPPS, intracranial haemangiopericytoma, metastatic cancer, liver metastases

\section{Introduction}

Intracranial haemangiopericytoma (HPC) is an exceedingly rare tumour which, despite its good prognosis, has a strong tendency to recur after treatment and to metastasise, particularly to the liver. The liver's ability to regenerate makes it a unique surgical target, and several surgical techniques have sought to exploit this regenerative capacity in the treatment of liver tumours. Associating liver partition with portal vein ligation for staged hepatectomy (ALPPS) is a new form of staged hepatectomy which combines portal vein occlusion with parenchymal division to encourage hypertrophy in the non-diseased hemi-liver. This allows the hypertrophied section of liver to compensate for the loss of function which occurs when the diseased hemi-liver is resected.

The following case report describes a patient who presented with metastatic liver disease 14 years after he was first diagnosed with intracranial HPC, and underwent an ALPPS procedure to treat his liver tumours. It details the aetiology, histopathology, management and prognosis of intracranial HPCs, as well as the discovery of ALPPS, its indications and contraindications. The risks and benefits of both neurosurgery for intracranial HPC, and ALPPS for liver tumours in general, are discussed in depth. The need for a pragmatic approach in treating uncommon conditions, whose treatment may not be well-documented in the literature, is also considered.

\section{Case presentation}

Mr X is a 64-year-old man whose medical story begins in March of 2003, when he suffered a seizure during the night. He presented to Accident and Emergency at the John Radcliffe Hospital where an MRI revealed a tumour in the left frontal lobe. Prior to this diagnosis, Mr X stated that he had felt perfectly well, although on further questioning he recalled short-lasting episodes of blurred vision and headaches. Mr underwent a left frontal lobe resection, at which point the tumour was identified histologically as a low-grade haemangiopericytoma. He then underwent adjuvant radiotherapy. Unfortunately the tumour recurred twice, in 2009 and 2015. On both of these occasions, Mr X again experienced seizures during the night, and both times he underwent surgical resection and radiotherapy. Since 2015, Mr X has been seizure-free, and his neurosurgery has not left him with any significant neurological issues; a recent full neurological assessment was completely normal. $\mathrm{Mr} \mathrm{X}$ has also not suffered any psychological problems due to his illness or its treatment, although he has been confused at times on the ward and in conversation his affect is flat.

Mr X's current admission was related to a development in late 2017, when routine blood tests revealed elevated bilirubin. Mr X denies experiencing any symptoms prior 
to this, except for the occasional bout of mild abdominal pain. A large mass was subsequently detected on the right side of the liver with PET-CT, as well as bibasal lung lesions. An MRI confirmed the presence of a large right-sided liver mass and lesions within segment III. Mr X was considered to be a good candidate for surgery as he was highly motivated and physically fit. He was referred to the care of the hepatopancreato-biliary (HPB) team at the Churchill hospital to determine if the liver metastases were potentially resectable.

Other than his cancer diagnosis, Mr X's medical history also includes GORD secondary to a hiatus hernia. In January 2015 he had a myocardial infarction and underwent coronary angioplasty and stenting. Since this time, $\mathrm{Mr}$ $\mathrm{X}$ reports that his exercise tolerance has been good; he jogs at least five miles three times per week. There is no family history of any diseases, including cancer, liver disease and cardiovascular disease. Mr X regularly takes aspirin, atorvastatin and ramipril for his cardiovascular disease. He also takes omeprazole for reflux and clobazam to prevent seizures. He has no known drug allergies. Mr X does not drink alcohol or use recreational drugs, and gave up smoking 35 years ago, before which time he smoked 25 cigarettes a day. He is a father of two living with his wife, and is in full-time work. He states that his ill health has not impacted upon his ability to work, nor has it affected his positive outlook or tendency to 'take things in his stride'.

Stage I of Mr X's ALPPS procedure was performed on 'day 0 , with no intra-operative complications. His recovery was largely smooth, although on day 1 post-op auscultation revealed reduced air entry bilaterally in his lung bases, which was attributed to bibasal atelectasis, confirmed on a chest X-ray. This was likely caused by shallow breathing due to diaphragmatic pain from the liver trauma. On examination 4 days post-op (stage I), Mr X was alert and comfortable in his chair. He was eating and drinking well, passing urine, and mobilising around the ward using a walking frame. He was receiving oxygen through a nasal cannula $(4 \mathrm{~L} / \mathrm{min})$ but showing no signs of respiratory distress. A coronal scar from his previous neurosurgery was visible on the top of Mr X's head. Two dressings were in place on Mr X's abdomen, one in the left hypochrondium and another on the right lumbar region, and the surgical scars beneath showed no signs of bleeding or infection. A Robinson's drain on the right-hand side was draining serous fluid. An abdominal exam was normal, save for some tenderness at the sites of the incisions. Mr X had not yet opened his bowels, so was prescribed lactulose and senna, and reported opening his bowels the next day.

A CT scan performed six days post-operatively revealed sufficient hypertrophy of the future liver remnant for stage II to be carried out on day 7 . In the days following the procedure $\mathrm{Mr} \mathrm{X}$ was apyrexial and his observations were within normal physiological ranges. He was also eating and drinking well and passing urine. However, liver function tests revealed a modest degree of liver failure, with a serum bilirubin of $140 \mathrm{~mol} / \mathrm{l}$ and alkaline phosphatase of 734IU/L. On examination, Mr X was jaundiced, with a distended abdomen due to ascites. However, he reported feeling completely well. He was prescribed spironolactone and albumin, and by day 13 his ascites had significantly improved. Mr X was also weaned off his PCA onto oral pain control, and his Robinson's drain was removed as there was no evidence of bila leakage. On day $18, \mathrm{Mr}$ X's serum bilirubin had halved to $71 \mathrm{~mol} / \mathrm{l}$, but his
ALP was further elevated at 1276IU/L, and he remained visibly jaundiced. Despite this, he was clinically well, and an ultrasound revealed no dilatation of the common bile duct. The decision was taken to discharge Mr X the next day, to be followed up in clinic later in the week.

\section{Discussion}

\section{Haemangiopericytoma and its treatment}

Haemangiopericytoma (HPC) is a rare malignant vascular tumour first described in 1942. It is thought to arise from pericytes surrounding capillaries and postcapillary venules [3]. HPCs tend to occur within soft tissues and can arise in many sites, including the skin, retroperitoneum, neck and oral cavity. Intracranial HPCs are rare, accounting for less than 1\% of all brain tumours, and are most often diagnosed between the ages of 38 and 42 [1]. Presenting symptoms vary according to tumour position, but many case reports describe similar symptoms to those experienced by Mr X, including blurred vision and headaches, as well as nausea and dizziness [2].

Intracranial HPCs are most often detected on MRI, but due to their similarity to meningioma in this modality a definitive diagnosis can only be made histologically following resection [8]. At a histological level, HPCs are characterised by their hypercellular appearance, 'staghorn sinusoid' vascular channels and dense reticulin networks. The WHO classifies HPC as a grade II tumour, although grade III anaplastic variants have also been described, associated with shorter overall survival [1] [4]. HPC is an aggressive tumour with a propensity for local recurrence after removal. It is not uncommon for tumours to recur twice after an initial resection [2] [3] [4], as was the case for Mr X. Metastasis is also very common; as in Mr X's case, many primary intracranial HPCs metastasis to liver and lung [2]. Other common sites of metastasis include bone and kidney, as well as pancreas in later-stage disease [4].

Due to the rarity of intracranial HPC, no randomised controlled trials have thus far been performed to determine the optimum treatment strategy. However, most authors agree on total resection where possible, followed by adjuvant radiotherapy, as a treatment of choice [1] [3] [4] [5], in line with the most recent UK guidelines on the treatment of soft tissue sarcomas [6]. Complete resection of the tumour is key in prolonging survival; Chen and colleagues report increased overall survival, as well as recurrence-free survival, in patients who underwent gross total resection (GTR) compared to those who had a subtotal tumour resection (STR), in agreement with Soyuer et al [7]. However, completeness of resection is limited by a number of factors, including tumour mass, vascularity and infiltration of adjacent structures such as sinuses, cranial nerves and brainstem [1]. In some patients deemed 'high-risk' with regard to the aforementioned factors, an STR is the only option if complications such as hemiparalysis, stroke and death are to be avoided [4]. One case series of 40 patients reported that GTR was only achieved in $48 \%$ of patients, with the rest receiving STR [9].

The role of radiotherapy is more controversial, although two case series have reported delayed tumour progression and recurrence in patients given adjuvant radiotherapy regardless of the completeness of resection [8] [9]. Radiotherapy is thought to alter the biology of any 
tumour cells remaining after resection, rendering them less proliferative. However, the use of radiotherapy too can be limited by tumour characteristics, in particular proximity to radiosensitive parts of the brain [9]

If treated, the prognosis for patients with intracranial HPCs is good. Chen et al. report a five year survival rate of $89 \%$, with $74 \%$ surviving for 10 years. They also note that the vast majority of patients function well enough to return to work after treatment; $95 \%$ of the 38 patients included in the study were back at work within three months [1]. Despite the success of combined resection and radiotherapy in extending survival, local tumour recurrence and metastasis remain common outcomes. As many as $80 \%$ of intracranial HPCs recur, and 20\% metastasise [2] [3] [9]. Regular surveillance in the form of whole-body imaging is therefore a key part of the long-term management of HPC.

\section{ALPPS}

Many extrahepatic primary cancers metastasise to the liver. It has been suggested that the liver is an attractive site for tumour seeding for two reasons; it receives blood from both the systemic and the portal circulation, and its fenestrated sinusoidal epithelium may allow for easy entry by tumour cells [10]. The need to resect liver metastases with a wide margin is limited by the risk of liver failure if insufficient normal tissue is left behind. Two-stage hepatectomy attempts to overcome this problem by manipulating the natural regenerative capacity of hepatocytes. The first stage of the procedure involves interrupting the portal blood supply to the diseased hemi-liver by ligation or embolization of the portal vein. If any tumour burden is present in the other half of the liver, this is cleared. The loss of blood supply to the diseased hemi-liver causes it to atrophy, with compensatory hypertrophy in the other hemi-liver [11]. CT or MRI volumetry is used to monitor the patient until this future liver remnant (FLR) is of sufficient size, at which point the second stage of the procedure is carried out, removing the disease hemi-liver and leaving the patient with an adequate FLR for normal function [12]. One of the chief drawbacks of this technique is the potential for tumour regrowth in the four- to eightweek wait between stages I and II; as a result two-stage hepatectomy is only curative in two-thirds of patients [11]

ALPPS (Associating Liver Partition and Portal vein ligation for Staged hepatectomy) is a surgical technique which increases the rate of hypertrophy of the FLR after stage I, allowing stage II to be completed within one or two weeks. The technique was created by chance in 2007 by German surgeon Dr Hans Schlitt during an attempted right hepatectomy. As the patient's FLR was insufficiently large to complete the procedure, Dr Schlitt performed a hepaticojejunostomy after portal vein ligation, dividing the liver along the falciform ligament. The FLR subsequently underwent rapid, massive hypertrophy and Dr Schlitt was able to resect the right hemi-liver several weeks later. His results were published in 2012, with a view to applying the same technique to other patients previously thought to be unsuitable for resection due to an insufficient FLR [13]. Stage I of ALPPS is similar to that of the conventional twostage hepatectomy, with the additional step of parenchymal transection between the diseased hemi-liver and the FLR. This step is thought to encourage hypertrophy in several ways. Firstly, it prevents the formation of neo-collaterals perfusing the segment whoseblood supplyhas been occluded
[14]. Secondly, the traumatic stimulus of parenchymal transection may provide additional hypertrophic drive to FLR hepatocytes in a way which is not yet understood [15].

The benefits of ALPPS compared to conventional twostage hepatectomy are clear. Much greater hypertrophy is achievable in a shorter interval; typically $61-93 \%$ over $9-14$ days [15]. The decreased interval between stages I and II reduces the potential for intervening tumour growth and the development of adhesions, leading to an improved capacity for complete resection even in patients in whom the FLR is small. Schadde and colleagues report that complete tumour resection was achieved in 83\% of ALPPS patients included in their study, compared to only $66 \%$ in the patients who underwent a traditional two-stage hepatectomy [12].

Greater resection rates with ALPPS come at a price of increased morbidity and mortality. Recent results from the European-African Hepato-Pancreato-Biliary Association (E-AHPBA) show post-operative mortality of $14.9 \%$, with morbidity as high as 50\% [15]. Chief among these complications is post-operative liver insufficiency, which occurs in $15-30 \%$ of patients [16], including $\mathrm{Mr} \mathrm{X}$, and whose cause is not fully understood. One current theory is that directing all the portal blood flow to a smaller section of the liver after ligation produces portal hypertension, which may in turn lead to obstruction within the sinusoids and endothelial dysfunction [15], so-called 'small-forsize' syndrome. Intra-operative Doppler ultrasonography to measure portal flow following ligation in stage I could allow surgeons to anticipate this process, and potentially intervene to reduce portal flow by splenectomy or splenorenal shunting [12]. Another possible explanation for post-operative liver failure is that, in some patients, hypertrophy of the FLR may not be accompanied by the same degree of functional increase, leading to insufficiency despite an apparently adequate volume [12]

There is also some concern about yet unknown long-term outcomes of ALPPS. Some authors have raised the possibility that liver trauma during transection could release tumour cells into the bloodstream or peritoneum to disseminate, or indeed that rapid FLR hypertrophy could induce equally rapid proliferation of any residual tumour cells. Either of these phenomena could result in an increased risk of tumour recurrence and metastasis post-ALPPS. Oncological followup studies have yet to be published for ALPPS cohorts [17], but preliminary results suggest that such concerns may be unfounded. Schadde et al. report similar 12-month tumour recurrence rates following ALPPS and traditional two-stage hepatectomy; $54 \%$ and $52 \%$ respectively [12].

The introduction of ALPPS has greatly improved the prognosis for many patients with primary liver tumours and metastatic liver disease. It is particularly well suited to those patients previously thought to be unresectable due to their insufficient FLR or the presence of bilateral tumours [15]. However, given the high mortality and morbidity associated with ALPPS, conventional two-stage hepatectomy remains the treatment of choice for most patients with mono-lobar malignancies and a large potential FLR [16]. One recent risk analysis based on results from the international ALPPS registry also indicates that patients aged 67 or older are unsuitable for ALPPS due to higher post-operative mortality in this group [17]. Moving forward, clear universal guidelines on the indications and contraindications for ALPPS, as well as improved techniques for measuring FLR function pre- 
stage II will be of great benefit in reducing the mortality and morbidity associated with this promising technique.

\section{Conflicts of interest}

None.

\section{Funding}

None.

\section{Consent}

The patient in question consented to the production and potential publication of this report. A signed consent form was submitted to the Medical School Offices of the John Radcliffe Hospital.

\section{References}

1.Chen, L. et al. Multimodal treatment and management strategies for intracranial haemangiopericytoma. J Clin Neurosci, 22, 718-25 (2014)

2. Chan, W. et al. 18F-FDG-PET-CT imaging findings of recurrent intracranial haemangiopericytoma with distant metastases. Br J Radiol, 83, 172-4 (2010)

3. Aleagha, M. et al. Recurrent intracranial haemangiopericytoma compatible to synovial sarcoma. J Pak Med Assoc, 60, 586-8 (2010)

4. Hiraide, T. et al. Pancreatic metastases of cerebellar hemangiopericytoma occurring 24 years after initial presentation: report of a case. Surg Today, 44, 558-63 (2014)

5. Damodaran, O. et al. Primary intracranial haemangiopericytoma: comparison of survival outcomes and metastatic potential in WHO grade II and III variants. J Clin Neurosci, 21, 1310-4 (2014)

6. Dangoor, A. et al. UK guidelines for the management of soft tissue sarcomas. Clin Sarcoma Res, 6, https://doi. org/10.1186/s13569-016-0060-4 (2016)

7. Soyuer, S. et al. Intracranial meningeal haemangiopericytoma: the role of radiotherapy: report of 29 cases and review of the literature. Cancer, 100, 1491-7 (2004)

8. Melone, A. et al. Intracranial haemangiopericytoma our experience in 30 years: a series of 43 cases and review of the literature. World Neurosurg, 81, 556-62 (2013)

9. Rutkowski, M. et al. Intracranial haemangiopericytoma: clinical experience and treatment considerations in a modern series of 40 adult patients. Cancer, 118, 1628-36 (2012)

10. Ananthakrishnan, A. et al. Epidemiology of primary and secondary liver cancers. Semin Intervent Radiol, 23, 47-63 (2006)

11. Vennarecci, G. et al. The ALPPS procedure : a surgical option for hepatocellular carcinoma with major vascular invasion. World J Surg, 38, 1498-1503 (2014)

12. Schadde, E. et al. ALPPS offers a better chance of complete resection in patients with primarily unresectable liver tumors compared with conventional staged hepatectomies: results of a multicenter analysis. World J Surg, 38, 1510-19 (2014)

13. Schnitzbauer, A. et al. Right portal vein ligation combined with in situ splitting induces rapid left lateral liver lobe hypertrophy enabling 2-staged extended right hepatic resection in small-for-size settings. Ann Surg, 255, 405-414 (2012)

14. Wilms, C. et al. Comparative study of portal vein embolization versus portal vein ligation for induction of hypertrophy of the future liver remnant using a mini-pig model. Ann Surg, 247, 825-34 (2008)

15. Bertens, K. et al. ALPPS: challenging the concept of unresectability - a systematic review. Int J Surg, 13, 280-7 (2015)

16. Ielpo, B. et al. Pearls and pitfalls on ALPPS procedure: new complications in a new technique. Updates Surg, 66, 159-61 (2014)

17. Linecker, M. et al. The ALPPS risk score: avoiding futile use of ALPPS. Ann Surg, 264, 763-771 (2016) 